\title{
Monotone and Concave Positive Solutions to a Boundary Value Problem for Higher-Order Fractional Differential Equation
}

\author{
Jinhua Wang, ${ }^{1,2}$ Hongjun Xiang, ${ }^{1}$ and Yuling Zhao ${ }^{2}$ \\ ${ }^{1}$ Department of Mathematics, Xiangnan University, Chenzhou 423000, China \\ ${ }^{2}$ School of Mathematics and Computational Science, Sun-Yat Sen University, Guangzhou 510275, China
}

Correspondence should be addressed to Hongjun Xiang, hunxhjxhj67@126.com

Received 31 March 2011; Accepted 14 July 2011

Academic Editor: K. Chang

Copyright (c) 2011 Jinhua Wang et al. This is an open access article distributed under the Creative Commons Attribution License, which permits unrestricted use, distribution, and reproduction in any medium, provided the original work is properly cited.

We consider boundary value problem for nonlinear fractional differential equation $D_{0+}^{\alpha} u(t)+$ $f(t, u(t))=0,0<t<1, n-1<\alpha \leq n, n>3, u(0)=u^{\prime}(1)=u^{\prime \prime}(0)=\cdots=u^{(n-1)}(0)=0$, where $D_{0+}^{\alpha}$ denotes the Caputo fractional derivative. By using fixed point theorem, we obtain some new results for the existence and multiplicity of solutions to a higher-order fractional boundary value problem. The interesting point lies in the fact that the solutions here are positive, monotone, and concave.

\section{Introduction}

In this paper, we deal with the following boundary value problem for higher-order fractional differential equation:

$$
\begin{gathered}
D_{0+}^{\alpha} u(t)+f(t, u(t))=0, \quad 0<t<1, n-1<\alpha \leqslant n, n>3, \\
u(0)=u^{\prime}(1)=u^{\prime \prime}(0)=\cdots=u^{(n-1)}(0)=0,
\end{gathered}
$$

where $D_{0+}^{\alpha}$ denotes the Caputo fractional derivative and $f:[0,1] \times[0,+\infty) \rightarrow[0,+\infty)$ is a real function. By using fixed point theorem, some sufficient conditions for existence and multiplicity of solutions to the above boundary value problem are obtained. Moreover, we will show that the solutions obtained here are positive, monotone, and concave.

Fractional differential equations are valuable tools in the modelling of many phenomena in various fields of science and engineering [1-5]. Due to their applications, fractional differential equations have gained considerable attentions and there has been a significant 
development in the study of existence of solutions, and positive solutions to boundary value problems for fractional differential equations (e.g., [6-9] and references therein).

Some papers are devoted to study the existence of solutions for higher-order fractional boundary value problem. Salem [10] investigated the existence of pseudosolutions for the nonlinear $m$-point boundary value problem of fractional type

$$
\begin{aligned}
& D^{\alpha} x(t)+q(t) f(t, x(t))=0, \quad 0<t<1, \alpha \in(n-1, n], n \geqslant 2, \\
& x(0)=x^{\prime}(0)=x^{\prime \prime}(0)=\cdots=x^{(n-2)}(0)=0, \quad x(1)=\sum_{i=1}^{m-2} \xi_{i} x\left(\eta_{i}\right) .
\end{aligned}
$$

Zhang [11] considered the existence of positive solutions to the singular boundary value problem for fractional differential equations

$$
\begin{gathered}
D_{0+}^{\alpha} u(t)+q(t) f\left(u, u^{\prime}, \ldots, u^{(n-2)}\right)=0, \quad 0<t<1, \alpha \in(n-1, n], n \geqslant 2, \\
u(0)=u^{\prime \prime}(0)=\cdots=u^{(n-2)}(0)=u^{(n-2)}(1)=0
\end{gathered}
$$

where $D_{0+}^{\alpha}$ is the Riemann-Liouville fractional derivative of order $\alpha$. In another paper, Zhang [9] studied the existence, multiplicity, and nonexistence of positive solutions for the following higher-order fractional boundary value problem:

$$
\begin{gathered}
D^{\alpha} u+\lambda h(t) f(u)=0, \quad 0<t<1, \alpha \in(n-1, n], n \geqslant 2, \\
u(1)=u^{\prime}(0)=\cdots=u^{(n-2)}(0)=u^{(n-1)}(0)=0,
\end{gathered}
$$

where $D^{\alpha}$ is the Caputo fractional derivative of order $\alpha$.

It seems that the authors of the papers only studied the existence of the solutions or positive solutions. No one consider the qualities of the solutions for boundary value problems of fractional differential equation. Motivated by all the above works, the aim of this paper is to study the monotone, concave, and positive solutions of a fractional differential equation.

The rest of the paper is organized as follows. In Section 2, we will introduce some lemmas and definitions which will be used later. In Section 3, the existence and multiplicity of positive solutions for the boundary value problem (1.1) will be discussed. In Section 4, examples are given to check our results.

\section{Basic Definitions and Preliminaries}

In this section, we introduce some necessary definitions and lemmas, which will be used in the proofs of our main results.

Definition 2.1 (see [12]). The integral

$$
I_{0+}^{\alpha} y(t)=\frac{1}{\Gamma(\alpha)} \int_{0}^{t}(t-s)^{\alpha-1} y(s) d s
$$

where $\alpha>0$ is called the Riemann-Liouville fractional integral of order $\alpha$. 
Definition 2.2 (see [12]). The Caputo fractional derivative for a function $y:(0, \infty) \rightarrow R$ can be written as

$$
D_{0+}^{\alpha} y(t)=\frac{1}{\Gamma(n-\alpha)} \int_{0}^{t}(t-s)^{n-\alpha-1} y^{(n)}(s) d s,
$$

where $n=[\alpha]+1,[\alpha]$ denotes the integer part of real number $\alpha$.

According to the definitions of fractional calculus, we can obtain that the fractional integral and the Caputo fractional derivative satisfy the following Lemma.

Lemma 2.3 (see [13]). Assume that $u \in C^{m}[0,1]$ and $\rho \in(m-1, m), m \in N$ and $v \in C^{1}[0,1]$. Then, for $t \in[0,1]$,
(a) $D_{0+}^{\rho} I_{0+}^{\rho} v(t)=v(t)$,
(b) $I_{0+}^{\rho} D_{0+}^{\rho} u(t)=u(t)-\sum_{k=0}^{m-1}\left(\left(u^{(k)}(0)\right) / k !\right) t^{k}$,
(c) $\lim _{t \rightarrow 0^{+}} D_{0+}^{\rho} u(t)=\lim _{t \rightarrow 0^{+}} I_{0+}^{\rho} u(t)=0$.

Definition 2.4. Let $E$ be a real Banach space over $R$. A nonempty convex closed set $p \subset E$ is said to be a cone, provided that

(a) $a u \in P$, for all $u \in P, a \geqslant 0$,

(b) $u,-u \in P$, implies $u=0$.

Definition 2.5. Let $E$ be a real Banach space and $P \subset E$ a cone. A function $\varphi: P \rightarrow[0, \infty)$ is called a nonnegative continuous concave functional if $\varphi$ is continuous and

$$
\varphi(\lambda x+(1-\lambda) y) \geqslant \lambda \varphi(x)+(1-\lambda) \varphi(y)
$$

for all $x, y \in P$ and $0 \leqslant \lambda \leqslant 1$.

Lemma 2.6 (see [14]). Let $E$ be a Banach space, $K \subseteq E$ a cone in $E$, and $\Omega_{1}, \Omega_{2}$ two bounded open subsets of $E$ with $0 \in \Omega_{1}$ and $\bar{\Omega}_{1} \subset \Omega_{2}$. Suppose that $T: K \cap\left(\bar{\Omega}_{2} \backslash \Omega_{1}\right) \rightarrow K$ is continuous and completely continuous such that either

(i) $\|T u\| \leqslant\|u\|$ for $u \in K \cap \partial \Omega_{1}, \quad\|T u\| \geqslant\|u\|$ for $u \in K \cap \partial \Omega_{2}$,

or

(ii) $\|T u\| \geqslant\|u\|$ for $u \in K \cap \partial \Omega_{1}, \quad\|T u\| \leqslant\|u\|$ for $u \in K \cap \partial \Omega_{2}$

holds. Then, $T$ has a fixed point in $K \cap\left(\bar{\Omega}_{2} \backslash \Omega_{1}\right)$.

Let $b, d, r>0$ be constants, $P_{r}=\{u \in P:\|u\|<r\}, P(\varphi, b, d)=\{u \in P: b \leqslant \varphi(u)$, $\|u\| \leqslant d\}$. 
Lemma 2.7 (see [15]). Let $P$ be a cone in real Banach space E. Let $T: \bar{P}_{c} \rightarrow \bar{P}_{c}$ be a completely continuous map and $\varphi$ a nonnegative continuous concave functional on $P$ such that $\varphi(u) \leqslant\|u\|$, for all $u \in \bar{P}_{c}$. Suppose that there exist constants $a, b, d$ with $0<a<b<d \leqslant c$ such that

$$
\begin{aligned}
& \text { (i) }\{u \in P(\varphi, b, d): \varphi(u)>b\} \neq \emptyset, \quad \varphi(T u)>b \quad \forall u \in P(\varphi, b, d), \\
& \text { (ii) }\|T u\|<a \quad \forall u \in \bar{P}_{a} \\
& \text { (iii) } \varphi(T u)>b, \quad \forall u \in P(\varphi, b, c) \text { with }\|T u\|>d .
\end{aligned}
$$

Then, $T$ has at least three fixed points $u_{1}, u_{2}$, and $u_{3}$ satisfying

$$
\left\|u_{1}\right\|<a, \quad b<\varphi\left(u_{2}\right), \quad\left\|u_{3}\right\|>a, \quad \varphi\left(u_{3}\right)<b .
$$

Lemma 2.8. Assume that $f(t, u) \in C([0,1] \times[0,+\infty),[0,+\infty))$, then $u \in C[0,1]$ be a solution of fractional boundary value problem (1.1) if and only if $u \in C[0,1]$ is a solution of integral equation

$$
u(t)=\int_{0}^{1} G(t, s) f(s, u(s)) d s,
$$

where

$$
G(t, s)= \begin{cases}\frac{(\alpha-1) t(1-s)^{\alpha-2}-(t-s)^{\alpha-1}}{\Gamma(\alpha)}, & 0 \leqslant s \leqslant t \leqslant 1 \\ \frac{(\alpha-1) t(1-s)^{\alpha-2}}{\Gamma(\alpha)}, & 0 \leqslant t \leqslant s \leqslant 1 .\end{cases}
$$

Proof. Firstly, we prove the necessity. Let $u \in C[0,1]$ is a solution of fractional boundary value problem (1.1). By Lemma 2.3, we have

$$
\begin{aligned}
u(t) & =-I_{0+}^{\alpha} f(t, u(t))+u(0)+u^{\prime}(0) t+\cdots+\frac{u^{(n-1)}(0)}{(n-1) !} t^{n-1} \\
& =-\frac{1}{\Gamma(\alpha)} \int_{0}^{t}(t-s)^{\alpha-1} f(s, u(s)) d s+u^{\prime}(0) t
\end{aligned}
$$

Therefore,

$$
u^{\prime}(t)=-\frac{1}{\Gamma(\alpha)} \int_{0}^{t}(\alpha-1)(t-s)^{\alpha-2} f(s, u(s)) d s+u^{\prime}(0)
$$

By the boundary value condition $u^{\prime}(1)=0$, we have

$$
u^{\prime}(0)=\frac{1}{\Gamma(\alpha)} \int_{0}^{1}(\alpha-1)(1-s)^{\alpha-2} f(s, u(s)) d s .
$$


Hence, we obtain

$$
\begin{aligned}
u(t) & =-\frac{1}{\Gamma(\alpha)} \int_{0}^{t}(t-s)^{\alpha-1} f(s, u(s)) d s+\frac{1}{\Gamma(\alpha)} \int_{0}^{1}(\alpha-1) t(1-s)^{\alpha-2} f(s, u(s)) d s \\
& =\int_{0}^{1} G(t, s) f(s, u(s)) d s .
\end{aligned}
$$

The necessity is proved.

Now, we prove the sufficiency. Let $u \in C[0,1]$ be a solution of integral equation (2.6). Then, we have

$$
\begin{aligned}
D_{0+}^{\alpha} u(t) & =-D_{0+}^{\alpha}\left(\int_{0}^{t} \frac{(t-s)^{\alpha-1}}{\Gamma(\alpha)} f(s, u(s)) d s\right)+\left(\int_{0}^{1} \frac{(\alpha-1)(1-s)^{\alpha-2}}{\Gamma(\alpha)} f(s, u(s)) d s\right) D_{0+}^{\alpha} t \\
& =-D_{0+}^{\alpha} I_{0+}^{\alpha} f(t, u(t))=-f(t, u(t)) .
\end{aligned}
$$

By direct computation, we obtain that

$$
u(0)=u^{\prime}(1)=u^{\prime \prime}(0)=\cdots=u^{(n-1)}(0)=0 .
$$

That is to say, $u$ is a solution of fractional boundary value problem (1.1). Thus, the sufficiency is proved.

Lemma 2.9. Let $f(t, u(t)) \in C([0,1] \times[0, \infty),[0, \infty))$. Then, the solution $u(t)$ of fractional boundary value problem (1.1) satisfies

(1) $u(t)$ is concave on $(0,1)$,

(2) $u(t) \geqslant 0$ is increasing for $t \in[0,1]$.

Proof. Suppose that $u(t)$ is a solution of fractional boundary value problem (1.1). By (2.11), we know that

$$
u(t)=-\frac{1}{\Gamma(\alpha)} \int_{0}^{t}(t-s)^{\alpha-1} f(s, u(s)) d s+\frac{1}{\Gamma(\alpha)} \int_{0}^{1}(\alpha-1) t(1-s)^{\alpha-2} f(s, u(s)) d s .
$$

Therefore,

$$
\begin{aligned}
& u^{\prime}(t)=-\frac{1}{\Gamma(\alpha)} \int_{0}^{t}(\alpha-1)(t-s)^{\alpha-2} f(s, u(s)) d s+\frac{1}{\Gamma(\alpha)} \int_{0}^{1}(\alpha-1)(1-s)^{\alpha-2} f(s, u(s)) d s, \\
& u^{\prime \prime}(t)=-\frac{1}{\Gamma(\alpha)} \int_{0}^{t}(\alpha-1)(\alpha-2)(t-s)^{\alpha-3} f(s, u(s)) d s \leqslant 0, \quad \text { for } t \in[0,1], n-1<\alpha \leqslant n, n>3,
\end{aligned}
$$

which implies that $u(t)$ is concave on $(0,1)$. The statement (1) is proved. 
Since $u^{\prime \prime}(t) \leqslant 0$, we know that $u^{\prime}(t)$ is nonincreasing. By $u^{\prime}(1)=0$, we have $u^{\prime}(t) \geqslant 0$, $t \in[0,1]$. Thus, $u(t)$ is increasing. Noting $u(0)=0$, we obtain that $u(t) \geqslant 0$ for $t \in[0,1]$. The statement (2) is proved.

Lemma 2.10. The Green's function $G(t, s)$, defined by (2.7), satisfies

(1) $\max _{0 \leqslant t \leqslant 1} G(t, s)=G(1, s), s \in[0,1]$,

(2) $G(t, s) \geqslant 0, t, s \in[0,1]$,

(3) $\min _{\xi \leqslant t \leqslant \eta} G(t, s) \geqslant \xi^{\alpha-1} G(1, s), s \in[0,1]$, for all $\xi, \eta \in(0,1), \xi<\eta$.

Proof. By (2.7), we have

$$
G_{t}^{\prime}(t, s)= \begin{cases}\frac{(\alpha-1)(1-s)^{\alpha-2}-(\alpha-1)(t-s)^{\alpha-2}}{\Gamma(\alpha)}, & 0 \leqslant s \leqslant t \leqslant 1, \\ \frac{(\alpha-1)(1-s)^{\alpha-2}}{\Gamma(\alpha)}, & 0 \leqslant t \leqslant s \leqslant 1 .\end{cases}
$$

It is clear that $G_{t}^{\prime}(t, s) \geqslant 0, t, s \in[0,1]$. Therefore, $G(t, s)$ is increasing respect to $t$ for $s \in[0,1]$. Thus, $\max _{0 \leqslant t \leqslant 1} G(t, s)=G(1, s)$. The statement (1) holds.

If $0 \leqslant t \leqslant s \leqslant 1$, then,

$$
G(t, s)=\frac{(\alpha-1) t(1-s)^{\alpha-2}}{\Gamma(\alpha)} \geqslant 0
$$

Since $G(t, s)$ is increasing respect to $t$ for $s \in[0,1]$, it is easy to see that $G(t, s) \geqslant 0$ for $0 \leqslant s \leqslant$ $t \leqslant 1$. We get the statement (2).

On the other hand, we have

$$
\begin{aligned}
\min _{\xi \leqslant t \leqslant \eta} G(t, s) & = \begin{cases}\frac{(\alpha-1) \xi(1-s)^{\alpha-2}-(\xi-s)^{\alpha-1}}{\Gamma(\alpha)}, & s \in[0, \xi], \\
\frac{(\alpha-1) \xi(1-s)^{\alpha-2}}{\Gamma(\alpha)}, & s \in[\xi, \eta], \\
\frac{(\alpha-1) \xi(1-s)^{\alpha-2}}{\Gamma(\alpha)}, & s \in[\eta, 1]\end{cases} \\
& = \begin{cases}\frac{(\alpha-1) \xi(1-s)^{\alpha-2}-(\xi-s)^{\alpha-1}}{\Gamma(\alpha)}, & s \in[0, \xi], \\
\frac{(\alpha-1) \xi(1-s)^{\alpha-2}}{\Gamma(\alpha)}, & s \in[\xi, 1] .\end{cases}
\end{aligned}
$$

If $s \in[0, \xi]$, then

$$
\begin{aligned}
(\alpha-1) \xi(1-s)^{\alpha-2}-(\xi-s)^{\alpha-1} & =\xi(\alpha-1)(1-s)^{\alpha-2}-\xi^{\alpha-1}(1-(s / \xi))^{\alpha-1} \\
& \geqslant \xi^{\alpha-1}(\alpha-1)(1-s)^{\alpha-2}-\xi^{\alpha-1}(1-s)^{\alpha-1} \\
& =\xi^{\alpha-1}\left[(\alpha-1)(1-s)^{\alpha-2}-(1-s)^{\alpha-1}\right] .
\end{aligned}
$$


If $s \in[\xi, 1]$, then

$$
\begin{aligned}
(\alpha-1) \xi(1-s)^{\alpha-2} & \geqslant(\alpha-1) \xi^{\alpha-1}(1-s)^{\alpha-2} \\
& \geqslant \xi^{\alpha-1}\left[(\alpha-1)(1-s)^{\alpha-2}-(1-s)^{\alpha-1}\right]
\end{aligned}
$$

Thus,

$$
\begin{aligned}
\min _{\xi \leqslant t \leqslant \eta} G(t, s) & \geqslant \xi^{\alpha-1} \frac{\left[(\alpha-1)(1-s)^{\alpha-2}-(1-s)^{\alpha-1}\right]}{\Gamma(\alpha)} \\
& =\xi^{\alpha-1} G(1, s), \quad s \in[0,1] .
\end{aligned}
$$

This yields the statement (3). The proof is finished.

\section{Main Results}

In this section, we establish the results for the existence and multiplicity of monotone and concave positive solutions for fractional boundary value problem (1.1).

Let $E=C[0,1]$ with $\|u\|=\max _{0 \leqslant t \leqslant 1}|u(t)|$. We define the cone $P \subset E$ by

$$
P=\left\{u \in E: u(t) \geqslant 0 \text { is concave on }[0,1], \min _{\xi \leqslant t \leqslant \eta} u(t) \geqslant \xi^{\alpha-1}\|u\|\right\} \text {. }
$$

And denote the operator $T$ by

$$
T u(t)=\int_{0}^{1} G(t, s) f(s, u(s)) d s
$$

Lemma 3.1. Assume that $f(t, u) \in C([0,1] \times[0,+\infty),[0,+\infty))$, then $T: P \rightarrow P$ is completely continuous.

Proof. In view of non-negativeness and continuity of $G(t, s)$ and $f(t, u(t))$, we know that the operator $T$ is continuous and $T u(t) \geqslant 0$, for $u \in P$.

By (2.16), we have

$$
\begin{aligned}
(T u)^{\prime \prime}(t) & =-\frac{1}{\Gamma(\alpha)} \int_{0}^{t}(\alpha-1)(\alpha-2)(t-s)^{\alpha-3} f(s, u(s)) d s \\
& \leqslant 0, \quad \text { for } t \in[0,1], n-1<\alpha \leqslant n, n>3
\end{aligned}
$$


Moreover, it follows from Lemma 2.10 that for $u \in P$,

$$
\begin{aligned}
\min _{\xi \leqslant t \leqslant \eta} T u(t) & =\min _{\xi \leqslant t \leqslant \eta} \int_{0}^{1} G(t, s) f(s, u(s)) d s \\
& \geqslant \xi^{\alpha-1} \int_{0}^{1} G(1, s) f(s, u(s)) d s \\
& =\xi^{\alpha-1}\|T u\| .
\end{aligned}
$$

Therefore, the operator $T: P \rightarrow P$ is well defined.

Assume that $\Omega \in P$ is bounded; that is, there exists a positive constant $M>0$ such that $\|u\| \leqslant M$ for all $u \in \Omega$. Let $N=\max _{0 \leqslant t \leqslant 1,\|u\| \leqslant M}|f(t, u(t))|+1$. For all $u \in \Omega$, we have

$$
|T u(t)|=\left|\int_{0}^{1} G(t, s) f(s, u(s)) d s\right| \leqslant N \int_{0}^{1} G(1, s) d s,
$$

which shows that $T(\Omega)$ is uniformly bounded.

In addition, for each $u \in \Omega, t_{1}, t_{2} \in[0,1]$ such that $t_{1}<t_{2}$, we have

$$
\begin{aligned}
\left|T u\left(t_{2}\right)-T u\left(t_{1}\right)\right|= & \left|\int_{0}^{1} G\left(t_{2}, s\right) f(s, u(s)) d s-\int_{0}^{1} G\left(t_{1}, s\right) f(s, u(s)) d s\right| \\
\leqslant & \left|\int_{0}^{t_{1}} \frac{\left(t_{1}-s\right)^{\alpha-1}-\left(t_{2}-s\right)^{\alpha-1}}{\Gamma(\alpha)} f(s, u(s)) d s-\int_{t_{1}}^{t_{2}} \frac{\left(t_{2}-s\right)^{\alpha-1}}{\Gamma(\alpha)} f(s, u(s)) d s\right| \\
& +\left|\int_{0}^{1} \frac{(\alpha-1)\left(t_{2}-t_{1}\right)(1-s)^{\alpha-2}}{\Gamma(\alpha)} f(s, u(s)) d s\right| \\
\leqslant & \frac{N}{\Gamma(\alpha+1)}\left(t_{2}^{\alpha}-t_{1}^{\alpha}\right)+\frac{N}{\Gamma(\alpha)}\left(t_{2}-t_{1}\right) .
\end{aligned}
$$

Thus, by the standard arguments, we obtain that $T(\Omega)$ is equicontinuous. The Arzela-Ascoli theorem implies that $T: P \rightarrow P$ is completely continuous. The proof is completed.

Let

$$
M_{1}=\int_{0}^{1} G(1, s) d s, \quad N_{1}=\xi^{\alpha-1} \int_{\xi}^{\eta} G(1, s) d s
$$

Theorem 3.2. Let $f(t, u) \in C([0,1] \times[0, \infty),[0, \infty))$. Assume that there exist two positive constants $r_{2}>r_{1}>0$ such that

$$
\begin{aligned}
& \left(H_{1}\right) f(t, u) \leq r_{2} / M_{1} \text { for }(t, u) \in[0,1] \times\left[0, r_{2}\right], \\
& \left(H_{2}\right) f(t, u) \geq r_{1} / N_{1} \text { for }(t, u) \in[0,1] \times\left[0, r_{1}\right] .
\end{aligned}
$$

Then, fractional boundary value problem (1.1) has at least one positive, increasing, and concave solution $u$ such that $r_{1} \leq\|u\| \leq r_{2}$. 
Proof. Lemmas 2.8 and 3.1 imply that $T: P \rightarrow P$ is completely continuous and fractional boundary problem (1.1) has a solution $u=u(t)$ if and only if $u$ satisfies the operator equation $u=T u$.

Let $\Omega_{1}:=\left\{u \in P:\|u\|<r_{1}\right\}$. By $\left(H_{2}\right)$, for $u \in \partial \Omega_{1}$ and $t \in[0,1]$, we have

$$
\begin{aligned}
T u(t) & =\int_{0}^{1} G(t, s) f(s, u(s)) d s \\
& \geqslant \frac{r_{1}}{N_{1}} \int_{\xi}^{\eta} \min _{\xi \leq t \leq \eta} G(t, s) d s \\
& \geqslant \frac{r_{1}}{N_{1}} \xi^{\alpha-1} \int_{\xi}^{\eta} G(1, s) d s=r_{1}=\|u\| .
\end{aligned}
$$

So,

$$
\|T u\| \geqslant\|u\|, \quad \text { for } u \in \partial \Omega_{1} .
$$

Let $\Omega_{2}:=\left\{u \in P:\|u\|<r_{2}\right\}$. For $u \in \partial \Omega_{2}$, and $t \in[0,1]$, it follows from $\left(H_{1}\right)$ that

$$
\begin{aligned}
\|T u(t)\| & =\max _{0 \leq t \leq 1}\left|\int_{0}^{1} G(t, s) f(s, u(s)) d s\right| \\
& \leqslant \frac{r_{2}}{M_{1}} \int_{0}^{1} G(1, s) d s \\
& =\frac{r_{2}}{M_{1}} \int_{0}^{1} G(1, s) d s=r_{2}=\|u\| .
\end{aligned}
$$

Lemma 2.6 implies that the fractional boundary value problem (1.1) has at least one positive solution $u$ such that $r_{1} \leq\|u\| \leq r_{2}$. By Lemma 2.9, the solution is also increasing and concave.

In order to use Lemma 2.7, we define the nonnegative continuous concave functional $\varphi$ by $\varphi(u)=\min _{\xi \leqslant t \leqslant \eta} u(t)$, for all $u \in P$.

Theorem 3.3. Suppose that $f(t, u) \in C([0,1] \times[0, \infty),[0, \infty))$ and there exist constants $0<a<$ $b<c$ such that the following conditions hold:

$$
\begin{aligned}
& \left(H_{3}\right) f(t, u)<\frac{a}{M_{1}} \text { for }(t, u) \in[0,1] \times[0, a], \\
& \left(H_{4}\right) f(t, u)>\frac{b}{N_{1}} \text { for }(t, u) \in[\xi, \eta] \times[b, c], \\
& \left(H_{5}\right) f(t, u) \leqslant \frac{c}{M_{1}} \text { for }(t, u) \in[0,1] \times[0, c] .
\end{aligned}
$$


Then, the fractional boundary problem (1.1) has at least three positive, increasing, and concave solutions $u_{1}, u_{2}$, and $u_{3}$ such that

$$
\begin{gathered}
\max _{0 \leqslant t \leqslant 1}\left|u_{1}(t)\right|<a, \quad b<\min _{\xi \leqslant t \leqslant \eta}\left|u_{2}(t)\right|<\max _{0 \leqslant t \leqslant 1}\left|u_{2}(t)\right| \leqslant c, \\
a<\max _{0 \leqslant t \leqslant 1}\left|u_{3}(t)\right| \leqslant c, \quad \min _{\xi \leqslant t \leqslant \eta}\left|u_{3}(t)\right|<b .
\end{gathered}
$$

Proof. By Lemmas 2.8 and 3.1, $T: P \rightarrow P$ is completely continuous, and fractional boundary value problem (1.1) has a solution $u=u(t)$ if and only if $u$ satisfies the operator equation $u=T u$.

First of all, we will prove the following assertions.

Assertion $3.4\left(T\left(\bar{P}_{c}\right) \subseteq \bar{P}_{c}\right.$ and $\left.T\left(\bar{P}_{a}\right) \subseteq \bar{P}_{a}\right)$. Firstly, Lemma 3.1 guarantees $T\left(\bar{P}_{c}\right) \subseteq P$. Secondly, for all $u \in \bar{P}_{c}$, we have $\|u\| \leqslant c$. By $\left(H_{5}\right)$,

$$
\begin{aligned}
\|T u(t)\| & =\max _{0 \leq t \leq 1}\left|\int_{0}^{1} G(t, s) f(s, u(s)) d s\right| \\
& \leqslant \frac{c}{M_{1}} \int_{0}^{1} G(1, s) d s \\
& =c
\end{aligned}
$$

which implies that $T\left(\bar{P}_{c}\right) \subseteq \bar{P}_{c}$. In the same way, $T\left(\bar{P}_{a}\right) \subseteq \bar{P}_{a}$.

Assertion $3.5(\{u \in P(\varphi, b, d) \mid \varphi(u)>b\} \neq \emptyset$, and $\varphi(T u)>b$, for all $u \in P(\varphi, b, d))$. Let $d=c$, $u=(b+c) / 2$. Then, $\|u\|<d$ and $\varphi(u)=\varphi((b+c) / 2)>b$. Consequently, $\{u \in P(\varphi, b, d) \mid$ $\varphi(u)>b\} \neq \emptyset$.

If $u \in P(\varphi, b, d)$, then from $\left(H_{4}\right)$ and Lemma 2.10, we obtain that

$$
\begin{aligned}
\varphi(T u) & =\min _{\xi \leqslant t \leqslant \eta} T u(t) \\
& \geqslant \int_{\xi}^{\eta} \min _{\xi \leqslant t \leqslant \eta} G(t, s) f(s, u(s)) d s \\
& >\frac{b}{N_{1}} \xi^{\alpha-1} \int_{\xi}^{\eta} G(1, s) d s \\
& =b .
\end{aligned}
$$

That is, $\varphi(T u)>b$, for all $u \in P(\varphi, b, d)$.

Assertion $3.6(\varphi(u)>b$, for all $u \in P(\varphi, b, c)$ with $\|u\|>d)$. If $u \in P(\varphi, b, c)$ and $\|T u\|>d=c$, similar to the above, we also have $\varphi(T u)>b$. 
Assertions $1 \sim 3$ imply that all conditions of Lemma 2.7 hold. Therefore, the fractional boundary value problem (1.1) has at least three positive solutions $u_{1}, u_{2}$, and $u_{3}$ satisfying

$$
\begin{gathered}
\max _{0 \leqslant t \leqslant 1}\left|u_{1}(t)\right|<a, \quad b<\min _{\xi \leqslant t \leqslant \eta}\left|u_{2}(t)\right|<\max _{0 \leqslant t \leqslant 1}\left|u_{2}(t)\right| \leqslant c, \\
a<\max _{0 \leqslant t \leqslant 1}\left|u_{3}(t)\right| \leqslant c, \quad \min _{\xi \leqslant t \leqslant \eta}\left|u_{3}(t)\right|<b .
\end{gathered}
$$

By Lemma 2.9, the positive solutions are also increasing and concave. The proof is completed.

\section{Example}

In this section, we will present some examples to show the effectiveness of our work.

Example 4.1. Consider the fractional boundary value problem

$$
\begin{gathered}
D_{0+}^{7 / 2} u(t)+t^{2}+\sqrt{u}+4=0, \quad 0<t<1, \\
u(0)=u^{\prime \prime}(0)=u^{\prime \prime \prime}(0)=u^{\prime}(1)=0 .
\end{gathered}
$$

Setting $\xi=1 / 2, \eta=3 / 4$, we obtain

$$
\begin{aligned}
M_{1} & =\int_{0}^{1} G(1, s) d s=\frac{(7 / 2)-1}{\Gamma(7 / 2)} \int_{0}^{1}(1-s)^{(7 / 2)-2} d s-\frac{1}{\Gamma(7 / 2)} \int_{0}^{1}(1-s)^{(7 / 2)-1} d s \\
& =\frac{1}{(5 / 2) \Gamma(5 / 2)}-\frac{1}{(7 / 2) \Gamma(7 / 2)} \approx 0.2149 \\
N_{1} & =\left(\frac{1}{2}\right)^{(7 / 2)-1} \int_{1 / 2}^{3 / 4} G(1, s) d s \\
& =\frac{(1 / 2)^{5 / 2}}{\Gamma(7 / 2)}\left(\int_{1 / 2}^{3 / 4} \frac{5}{2}(1-s)^{(7 / 2)-2} d s-\int_{1 / 2}^{3 / 4}(1-s)^{(7 / 2)-1} d s\right) \approx 0.0065 .
\end{aligned}
$$

Let $r_{1}=1 / 40, r_{2}=4$. We have

$$
\begin{aligned}
& f(t, u)=t^{2}+\sqrt{u}+4 \leqslant \frac{r_{2}}{M_{1}} \approx 18.613, \quad \text { for }(t, u) \in[0,1] \times[0,4], \\
& f(t, u)=t^{2}+\sqrt{u}+4 \geqslant \frac{r_{1}}{N_{1}} \approx 3.846, \quad \text { for }(t, u) \in[0,1] \times\left[0, \frac{1}{40}\right] .
\end{aligned}
$$

Theorem 3.2 implies that fractional boundary value problem (4.1) has at least one positive, increasing, and concave solution. The approximate solution is obtained by the Adams-type predictor-corrector method [16], which is displayed in Figure 1 for the step size $h=0.01$. 


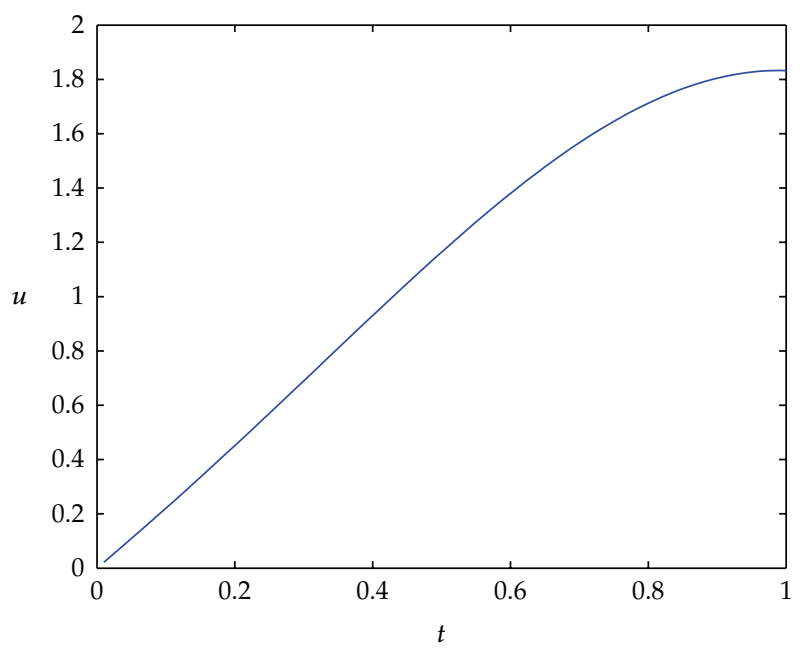

Figure 1: Transient response of state variable $u(t)$.

Example 4.2. Consider the fractional boundary value problem

$$
\begin{gathered}
D_{0+}^{7 / 2} u(t)+f(t, u)=0, \quad 0<t<1 \\
u(0)=u^{\prime \prime}(0)=u^{\prime \prime \prime}(0)=u^{\prime}(1)=0
\end{gathered}
$$

where

$$
f(t, u)= \begin{cases}\frac{t}{10}+155 u^{3}, & 0 \leqslant u \leqslant 1, t \in[0,1] \\ \frac{t}{10}+u+154, & u>1, t \in[0,1]\end{cases}
$$

Setting $\xi=1 / 2, \eta=3 / 4$, we know that $M_{1} \approx 0.2149, N_{1} \approx 0.0065$. Choosing $a=$ $1 / 10, b=1, c=45$, we obtain that

$$
\begin{aligned}
& f(t, u)=\frac{t}{10}+155 u^{3}<\frac{a}{M_{1}} \approx 0.466 \text { for }(t, u) \in[0,1] \times\left[0, \frac{1}{10}\right], \\
& f(t, u)=\frac{t}{10}+u+154>\frac{b}{N_{1}} \approx 153.846 \text { for }(t, u) \in\left[\frac{1}{2}, \frac{3}{4}\right] \times[1,45], \\
& f(t, u)=\frac{t}{10}+u+154<\frac{c}{M_{1}} \approx 209.4 \text { for }(t, u) \in[0,1] \times[0,45] .
\end{aligned}
$$




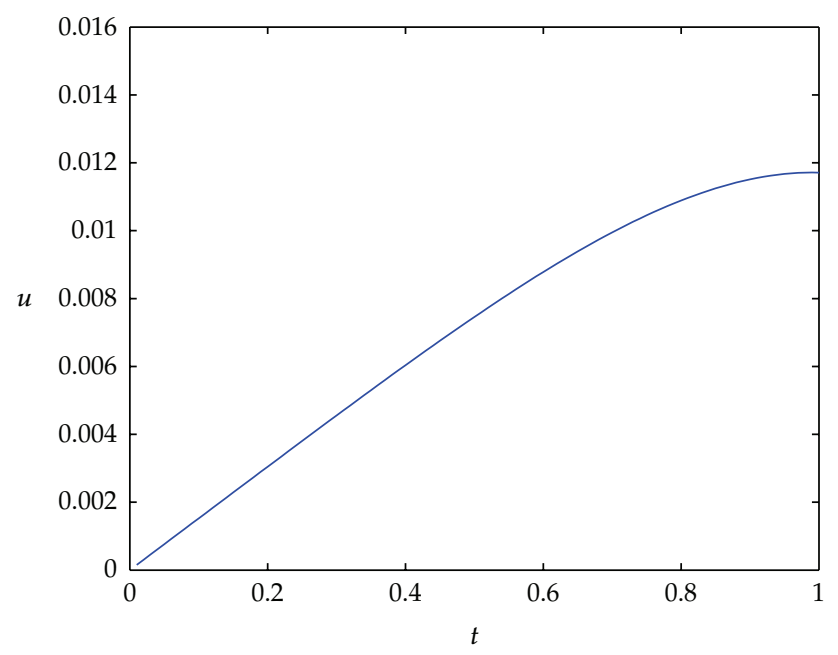

Figure 2: Transient response of state variable $u(t)$.

Theorem 3.3 implies that fractional boundary value problem (4.4) has three positive, increasing, and concave solutions such that

$$
\begin{gathered}
\max _{0 \leqslant t \leqslant 1}\left|u_{1}(t)\right|<\frac{1}{10}, \quad 1<\min _{1 / 2 \leqslant t \leqslant 3 / 4}\left|u_{2}(t)\right|<\max _{0 \leqslant t \leqslant 1}\left|u_{2}(t)\right| \leqslant 45, \\
\frac{1}{10}<\max _{0 \leqslant t \leqslant 1}\left|u_{3}(t)\right| \leqslant 45, \quad \min _{1 / 2 \leqslant t \leqslant 3 / 4}\left|u_{3}(t)\right|<1 .
\end{gathered}
$$

For numerical simulation case 1, Figure 2 depicts the phase responses state variables of $u(t)$ with the step size $h=0.01$.

\section{Acknowledgments}

This work was jointly supported by Natural Science Foundation of China (no. 10871214), Natural Science Foundation of Hunan Provincial under Grants nos. 11JJ3005, 10JJ6007, and 2010GK3008.

\section{References}

[1] L. Gaul, P. Klein, and S. Kemple, "Damping description involving fractional operators," Mechanical Systems and Signal Processing, vol. 5, no. 2, pp. 81-88, 1991.

[2] W. G. Glockle and T. F. Nonnenmacher, "A fractional calculus approach to self-similar protein dynamics," Biophysical Journal, vol. 68, no. 1, pp. 46-53, 1995.

[3] R. Metzler, W. Schick, H. G. Kilian, and T. F. Nonnenmacher, "Relaxation in filled polymers: a fractional calculus approach," Journal of Chemical Physics, vol. 103, no. 16, pp. 7180-7186, 1995.

[4] R. Metzler and J. Klafter, "The random walk's guide to anomalous diffusion: a fractional dynamics approach," Physics Reports, vol. 339, no. 1, pp. 1-77, 2000.

[5] F. Mainardi, Fractional Calculus and Waves in Linear Viscoelasticity, Imperial College Press, London, UK, 2010. 
[6] A. Arara, M. Benchohra, N. Hamidi, and J. J. Nieto, "Fractional order differential equations on an unbounded domain," Nonlinear Analysis, vol. 72, no. 2, pp. 580-586, 2010.

[7] S. Liang and J. Zhang, "Positive solutions for boundary value problems of nonlinear fractional differential equation," Nonlinear Analysis, vol. 71, no. 11, pp. 5545-5550, 2009.

[8] J. H. Wang, H. Xiang, and Z. Liu, "Positive solutions for three-point boundary value problems of nonlinear fractional differential equations with $p$-Laplacian," Far East Journal of Applied Mathematics, vol. 37, no. 1, pp. 33-47, 2009.

[9] S. Q. Zhang, "Existence results of positive solutions to boundary value problem for fractional differential equation," Positivity, vol. 13, no. 3, pp. 583-599, 2009.

[10] H. A. H. Salem, "On the fractional order m-point boundary value problem in reflexive Banach spaces and weak topologies," Journal of Computational and Applied Mathematics, vol. 224, no. 2, pp. 565-572, 2009.

[11] S. Q. Zhang, "Positive solutions to singular boundary value problem for nonlinear fractional differential equation," Computers E Mathematics with Applications, vol. 59, no. 3, pp. 1300-1309, 2010.

[12] I. Podlubny, Fractional Differential Equations, vol. 198 of Mathematics in Science and Engineering, Academic Press, San Diego, Calif, USA, 1999.

[13] N. Kosmatov, "Integral equations and initial value problems for nonlinear differential equations of fractional order," Nonlinear Analysis, vol. 70, no. 7, pp. 2521-2529, 2009.

[14] M. A. Krasnoselskii, Positive Solutions of Operator Equations, Noordhoff, Groningen, The Netherlands, 1964.

[15] R. W. Leggett and L. R. Williams, "Multiple positive fixed points of nonlinear operators on ordered Banach spaces," Indiana University Mathematics Journal, vol. 28, no. 4, pp. 673-688, 1979.

[16] E. Ahmed, A. M. A. El-Sayed, A. E. M. El-Mesiry, and H. A. A. El-Saka, "Numerical solution for the fractional replicator equation," International Journal of Modern Physics C, vol. 16, no. 7, pp. 1-9, 2005. 


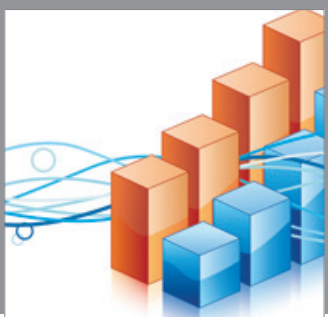

Advances in

Operations Research

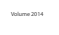

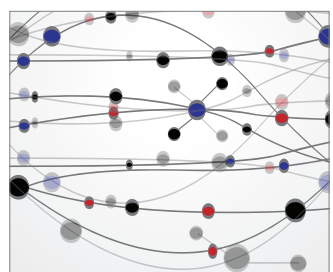

\section{The Scientific} World Journal
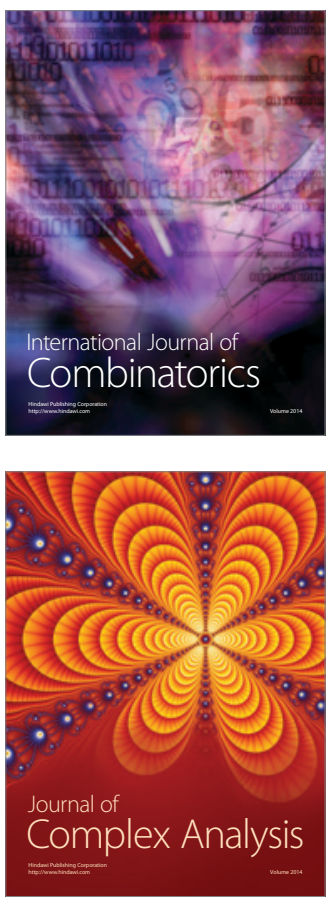

International Journal of

Mathematics and

Mathematical

Sciences
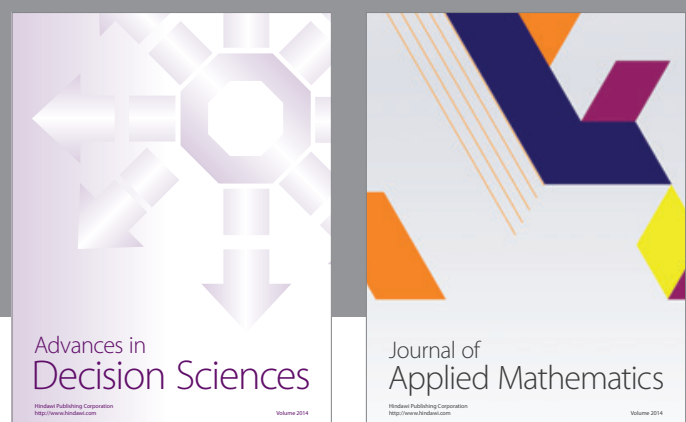

Journal of

Applied Mathematics
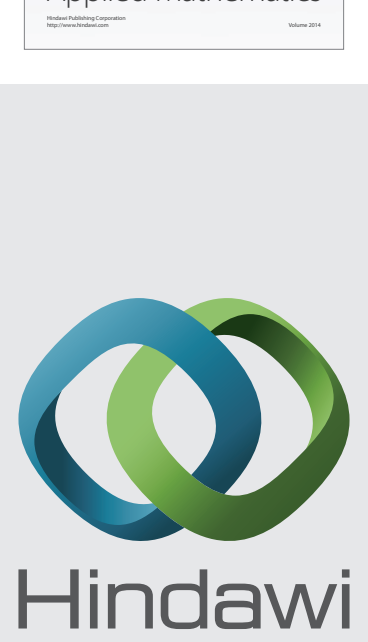

Submit your manuscripts at http://www.hindawi.com
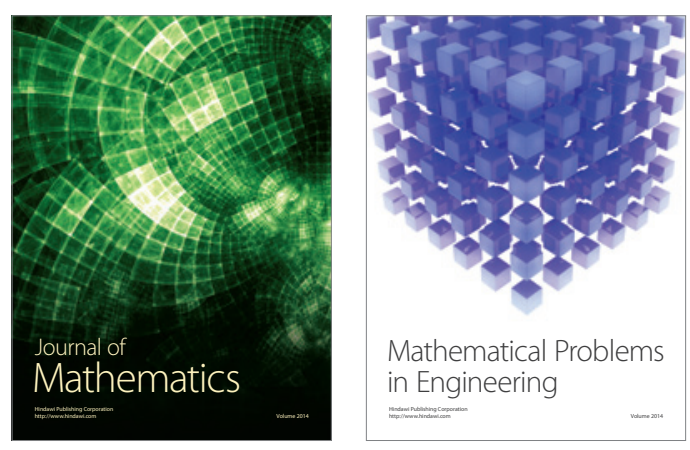

Mathematical Problems in Engineering
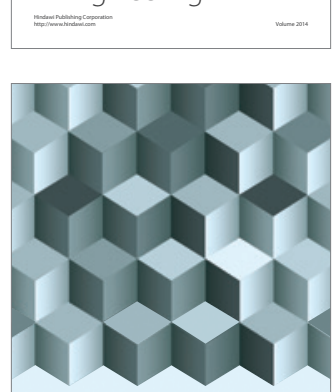

Journal of

Function Spaces
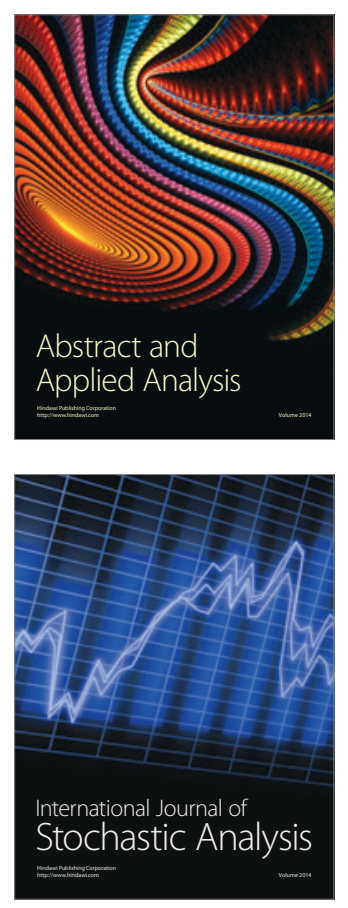

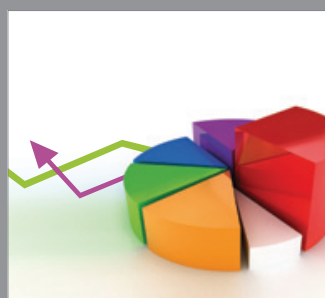

ournal of

Probability and Statistics

Promensencen
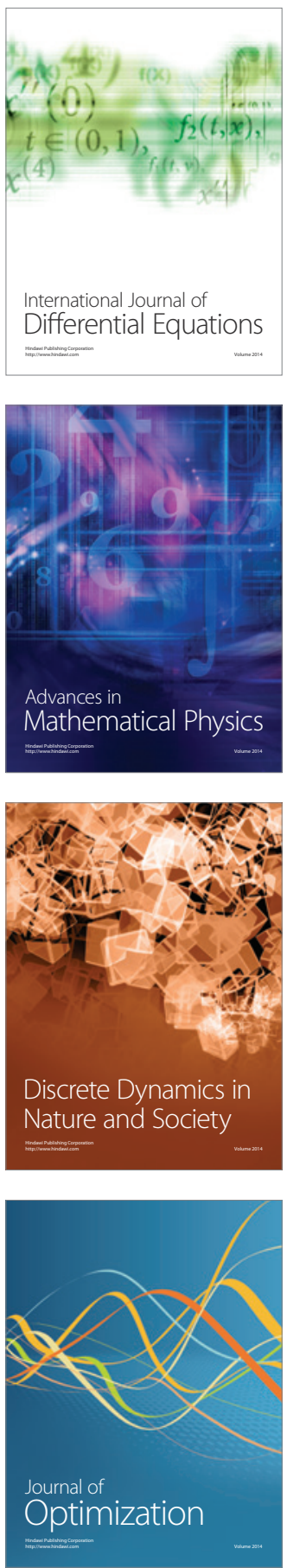